\title{
MEMS-Based Mechanical Spectrum Analyzer
}

\author{
Luis Alexandre Rocha, Edmond Cretu, and Reinoud F. Wolffenbuttel
}

\begin{abstract}
An ac-operated capacitive accelerometer with electrostatic force feedback is employed for direct mechanical spectrum analysis. Force feedback acts as a means for electronic modulation of the spring constant of the suspension and thus for enhancing the sensitivity. Therefore, a suitable electrostatic time-varying actuation can be used to make the accelerometer selectively sensitive to a coherent frequency component of a mechanical vibration. By sweeping the frequency of the drive voltage over a selected range, the mechanical (vibration) spectrum is analyzed in the mechanical domain. An inverted pendulum type of accelerometer structure has been fabricated in silicon using micromachining techniques and operated using electrostatic momentum feedback. The spectral performance is comparable to fast Fourier transform based systems for spectral analysis on a time series supplied by a conventional accelerometer, while the overall system features a reduced complexity and reduced power consumption.
\end{abstract}

Index Terms-Accelerometers, microelectromechanical systems (MEMS), spectrum analyzer, vibration analyzer.

\section{INTRODUCTION}

$\mathbf{M}$ ANY applications require processing of the spectral content of the input mechanical signal. Conventional techniques for condition monitoring of mechanical machines based on vibration monitoring for early failure detection generally use algorithms operating in the frequency domain, rather than in the time domain [1]. The complexity and inaccessibility of the systems being monitored ask for permanently mounted vibration analysis systems. Low cost and small size monitoring systems would ensure a rapid penetration in a range of applications [2], [3].

From a sensing perspective, two main approaches are currently used.

1) Vibration analysis in the electrical domain, which is conventionally implemented in a straightforward way by having an accelerometer to measure a time series and a digital signal processor (DSP) to perform a fast Fourier transform (FFT) [4]. However, the DSP die size and power dissipation make such a solution difficult to implement in a microinstrument.

2) An array of tuned resonators measures the mechanical vibration. Each resonator is tuned on a specific frequency, so several spectral lines are obtained in parallel [4]. The flexibility of the scheme is reduced compared with the first approach, but allows an improvement of the signal-to-noise ratio for the chosen resonant frequencies.

Manuscript received June 15, 2003; revised September 8, 2004. This work was supported by the Netherlands Technology Foundation under Grant DEL55.3733.

L. A. Rocha and R. F. Wolffenbuttel are with the Department for Microelectronics, Delft University of Technology, 2628 CD Delft, The Netherlands.

E. Cretu is with Melexis, B-3980 Tessenderlo, Belgium.

Digital Object Identifier 10.1109/TIM.2005.847168
In this paper, an alternative approach is pursued, in which a micromachined capacitive accelerometer structure is operated using electrostatic feedback with an ac drive component introduced in the loop [5]. This is basically an extension of the electrostatic force feedback in servo-operated accelerometers with a dc voltage used for setting the loop gain [6]. Capacitive accelerometers with electrostatic force feedback are widely employed, because of performance and robustness. The force feedback acts as a means for electronic modulation of the spring constant of the suspension, "negative spring effect," and thus enables electronic control of sensitivity and resonance frequency. Commonly, the electrostatic feedback is employed for null-based acceleration measurement and provides an output signal in the form of a duty-cycle modulation, frequency modulation, or a sigma-delta bitstream format.

Extending this principle by applying an ac component in the excitation voltage enables the realization of a mechanical spectrum analyzer with frequency selectivity directly in the mechanical domain. A wide-band accelerometer can be made selectively sensitive to a narrow frequency component of a mechanical vibration that is coherent with the electrically driving forces. By sweeping the frequency of the electrical signal over a selected range, the frequency components of the mechanical vibration are obtained sequentially with a resolution determined by the details of the feedback loop.

A mechanical spectrum analyzer based on an accelerometer operated using electrostatic momentum feedback is presented and analyzed in this paper. First experimental results show that the technique compares well with more conventional techniques. The main advantages of this approach are the low cost, low power, reduced size, and system complexity, which makes the system very suitable in low power applications [7].

\section{DEVICE DESCRIPTION}

Device operation is such that it provides the frequency components sequentially, rather than a time series of the acceleration. The principle is universal and could be applied to any type of accelerometer, provided that the structure allows for electrostatic driving. In this paper, a clamped inverted balance accelerometer structure is used.

The conceptual device is the conventional single-sided clamped inverted pendulum in the gravitational field, as shown in Fig. 1. The weight of the seismic mass $m$ is assumed to be concentrated at the top. The clamped beam has elastic properties only, and is considered massless. In the absence of any horizontal acceleration component, the vertical position is an equilibrium one. Any horizontal inertial force causes a displacement from the vertical position $\delta$ until the reaction developed at the clamping point equilibrates the external action. Without a gravitational field, this external action is solely 


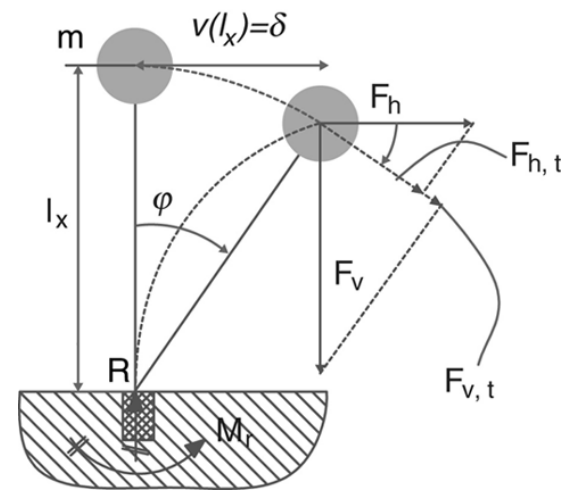

Fig. 1. Operating principle of the inverted pendulum in the gravitational field.

determined by the horizontal force; however, in the presence of a gravitational field, the effect is magnified by the force $\mathrm{F}_{\mathrm{vt}}$ (Fig. 1) and thus yields a larger equilibrium deflection.

The same principle remains valid if the vertical gravitational field is replaced with an electrostatic field. The added value is in the electronic control of this field, and thus the potential of dynamic tuning of the feedback effect.

In the micro device under investigation, the DC gravitational field is replaced by an ac electrostatic field. The frequency of the ac drive voltage determines the frequency for which this positive feedback actually takes place. The actual structure fabricated in silicon using micromachining techniques is planar, rather than vertical, and is shown in Fig. 2. As the gravitational forces are negligible as compared to the electrostatic forces at the given dimensions, this change in device orientation has no practical implication on the operation. The anchor point is shown in the upper right corner, and interdigitated finger electrodes are available for electrostatic actuation and capacitive detection of displacement. Stoppers are included on either side of the freestanding tip to limit the lateral displacement range. Prototypes have been realized in the Bosch epipoly process [8]. ${ }^{1}$ Basically, an $11-\mu \mathrm{m}$-thick polysilicon layer is patterned and released in a surface-micromachining-alike process.

\section{OPERATING PRINCIPLE}

The key element is the dependence of the electrostatic force on the displacement, which can be linearized for displacements that are small relative to the gap size. Considering a generic accelerometer with an input inertial acceleration and a common-mode voltage far below the resonant frequency of the accelerometer, the quasi-static equivalent model presented in Fig. 3 is obtained.

The positive electrostatic force feedback induced by dc voltage is conventionally used for the electronic introduction of a negative compliance in capacitive servo accelerometers. The overall effect is a reduced stiffness of the physically available suspension. The stiffness of the suspension in the nonpowered condition can be large, greatly adding to device reliability. The tuning of the spring constant allows for electronic tuning of the sensitivity or the resonant frequency of the structure.

A more advanced use of the structural coupling between the mechanical and electrostatic fields results in the case of a time-

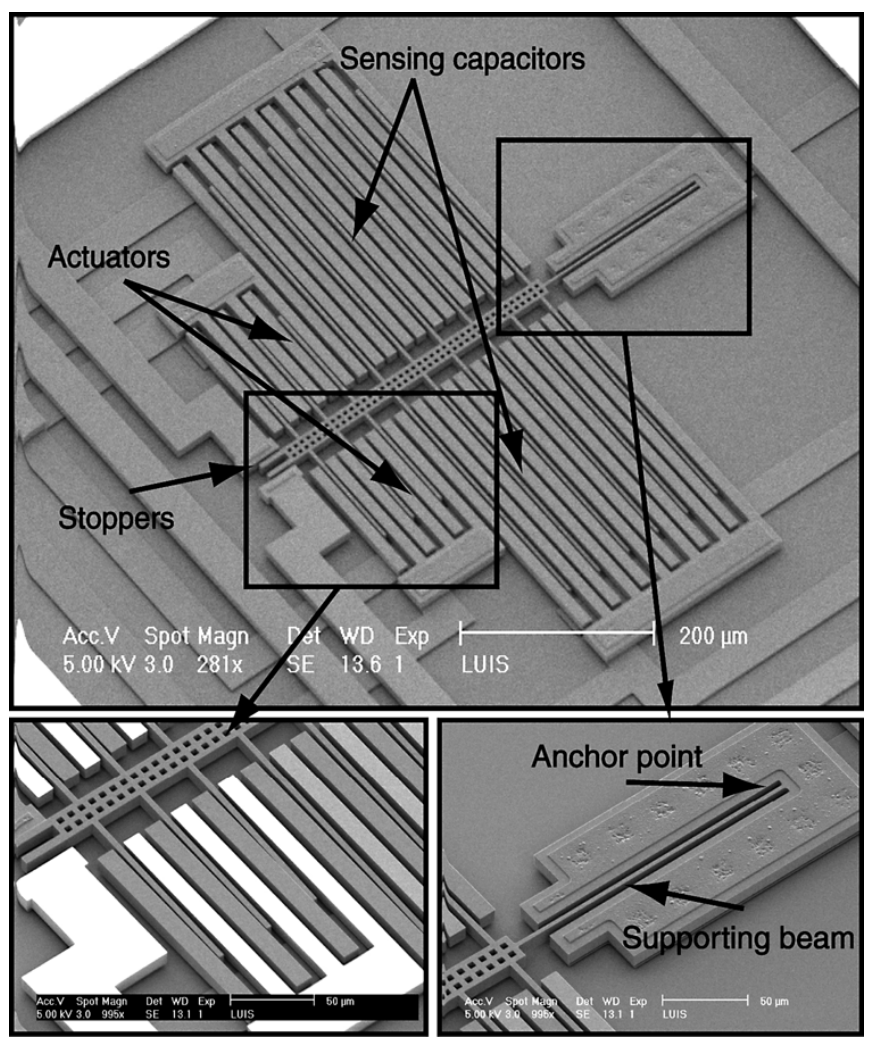

Fig. 2. Photograph showing a detailed view of the tip of the fabricated microstructure.

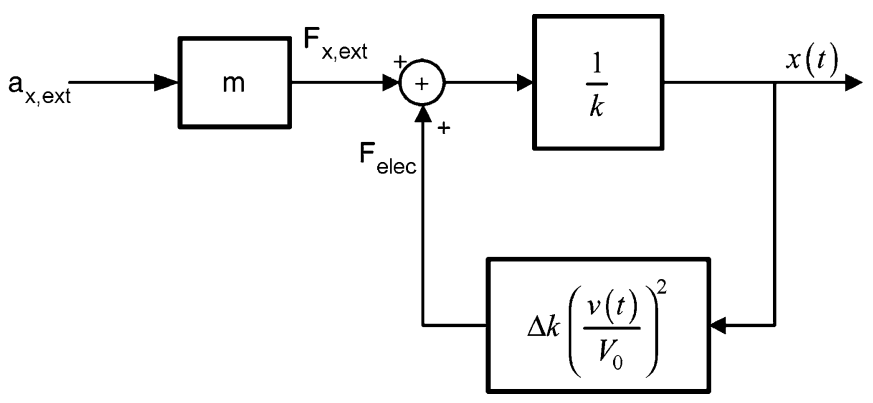

Fig. 3. Quasi-static model of common-mode actuated accelerometer.

varying actuation voltage. The voltage-dependent gain suggests the possibility of applying a correlation method, in order to extract a desired spectral component $\mathrm{X}(\omega)$ from the input mechanical acceleration signal

$$
\begin{aligned}
X(\omega)= & \underbrace{}_{T \rightarrow \infty} \frac{2}{T} \int_{0}^{T} x(t) e^{-j \omega t} d t \\
= & \underbrace{\lim _{T \rightarrow \infty} \frac{2}{T} \int_{0}^{T} x(t) \cos (\omega t) d t}_{X_{c}(\omega)} \\
& -\underbrace{\lim _{T \rightarrow \infty} \frac{2}{T} \int_{0}^{T} x(t) \sin (\omega t) d t}_{X_{s}(\omega)} .
\end{aligned}
$$

\footnotetext{
${ }^{1}$ http://www.europractice.bosch.com/en/start/index.htm.
} 


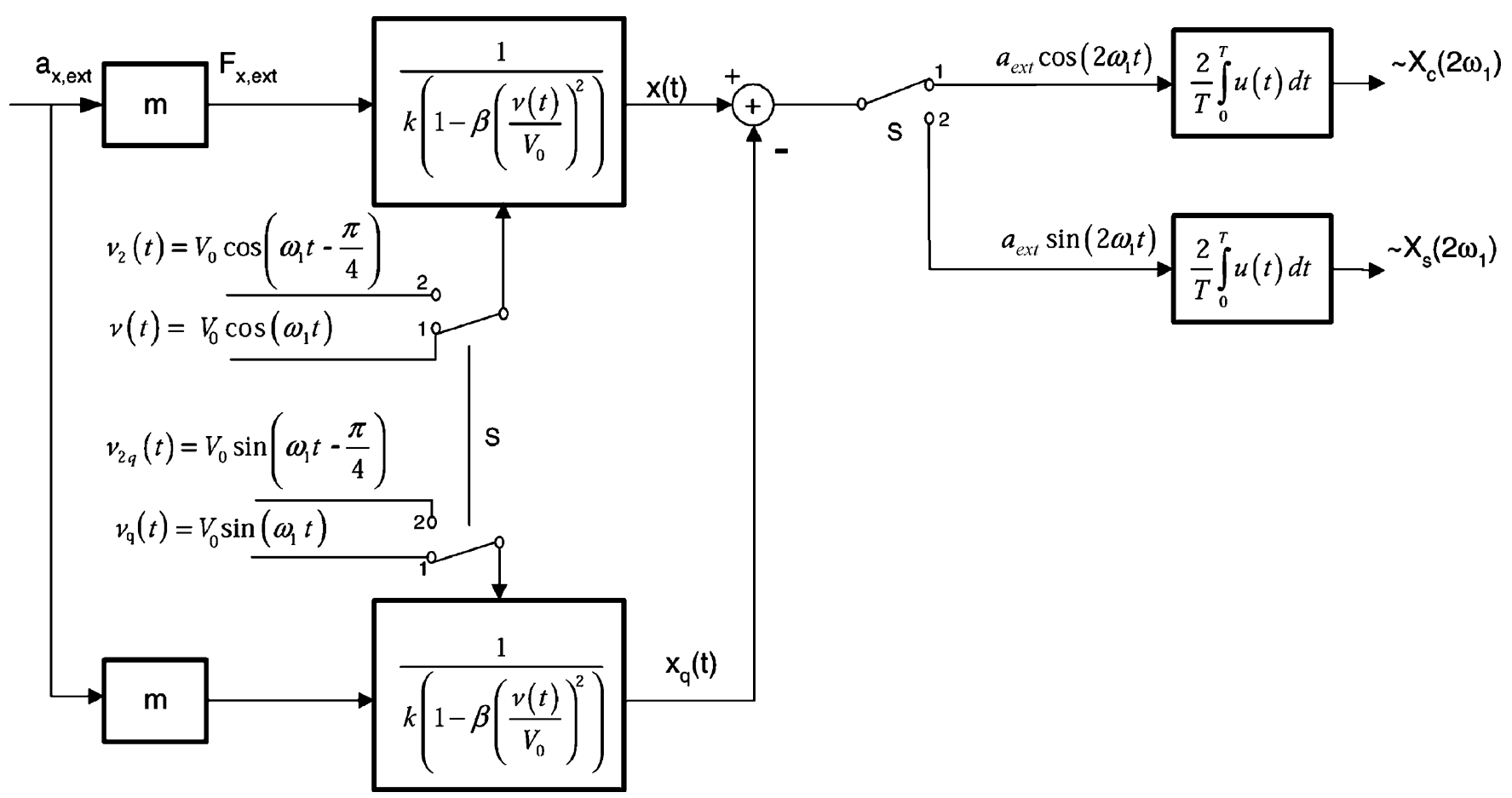

Fig. 4. Block diagram of the mechanical spectrum extraction scheme.

The basic system concept is shown schematically in Fig. 4, in the case of quasi-static operating mode (actuation frequencies lower than the mechanical resonance frequency). This is basically an extension of the chopper combined with coherent detector that is often used in instrumentation and measurement.

The main difference, as compared to the conventional coherent detector preceded by a chopper (AM modulator), is that the modulation parameter (actuation voltage) is present, not in a forward, but in the feedback path. Moreover, this parameter is squared. This leads to a transfer function of the system that can be written, for the general case as (Fig. 3)

$$
x(t)=F_{x, \mathrm{ext}} \frac{1}{k-\Delta k\left(\frac{v(t)}{V_{0}}\right)^{2}}=x_{0} \frac{1}{1-\beta\left(\frac{v(t)}{V_{0}}\right)^{2}}
$$

where $k$ is the mechanical spring of the device, $\Delta k$ is the change in the spring caused by the feedback path, $\beta=\Delta k / k$ is the nondimensional modulation constant (depends on the design and on the feedback path characteristics), $x_{0}=F_{\text {ext }} / k$ is the displacement caused by an external force (external acceleration), and $V_{0}$ is a parameter setting the gain and operation point. In the case of a harmonic actuation voltage, $v(t)=V_{0} \cos \left(\omega_{1} t\right)$, and according to (2), the gain introduced by the feedback path will vary periodically with a frequency $\omega_{2}=2 \omega_{1}$

$$
\frac{x(t)}{x_{0}(t)} \stackrel{\wedge}{=}(t)=\frac{1}{\left(1-\frac{\beta}{2}\right)} \frac{1}{\left(1-\frac{\beta}{2-\beta} \cos \left(\omega_{2} t\right)\right)} .
$$

Since the gain $G(t)$ is periodic, a discrete Fourier series decomposition can be made. Due to the symmetry (even function), only the cosine coefficients appear in the Fourier series. Fig. 5 shows a bar diagram of the coefficients of the Fourier series for a modulation factor $\beta=0.7$. With the exception of the dc component $g[0]$ and the higher order harmonics, the transfer function comes

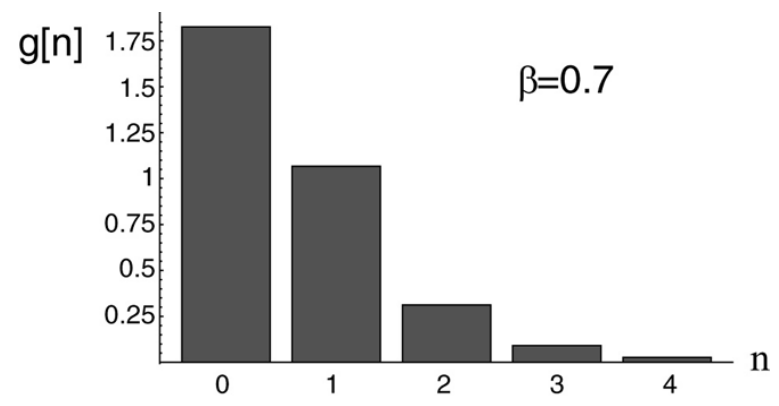

Fig. 5. Fourier coefficients for harmonic voltage actuation.

close to the concept of multiplying the input mechanical acceleration with an electrically controlled signal.

The disadvantage of the nonlinear feedback and of placing the control voltage in the feedback path is the presence of dc and higher harmonics. A substantial improvement results from the use of a twin-accelerometer structure, as schematically shown in Fig. 4. In this case, the equivalent time-varying gain is

$$
\frac{x(t)}{x_{0}(t)} \triangleq G_{q}(t)=\frac{\beta}{1-\beta+\frac{\beta^{2}}{8}} \frac{\cos \left(\omega_{2} t\right)}{1-\frac{\beta^{2}}{8-8 \beta-\beta^{2}} \cos \left(2 \omega_{2} t\right)} .
$$

The two identical accelerometers are actuated in quadrature (with $\pi / 2$ phase shift) and, as in a capacitor the electrostatic force is given by the square of the voltage, the initial phase shifts are transformed. With this transformation in the phase shift, all the even harmonics of the transfer function, including the dc component, are eliminated after subtraction of the output signals of the two accelerometers. Consequently, the distortion is significantly lower as compared to classic harmonic modulation in the case of a single channel scheme.

A comparison of the time-varying gain for the quadrature actuation with the simple harmonic actuation is shown in Fig. 6 


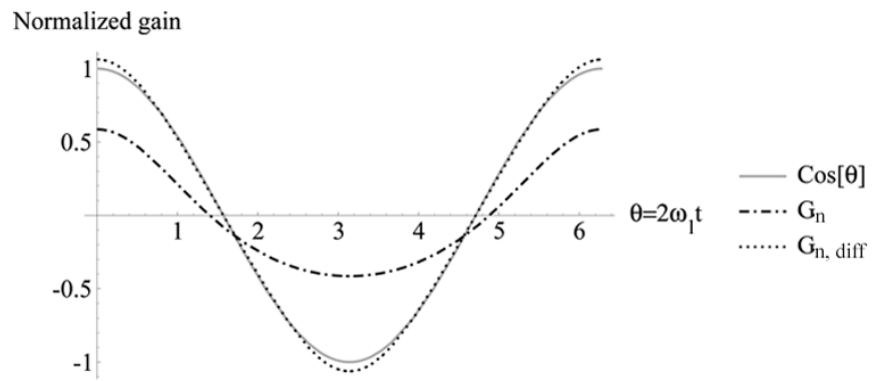

Fig. 6. Normalized gains compared with a pure harmonic function.

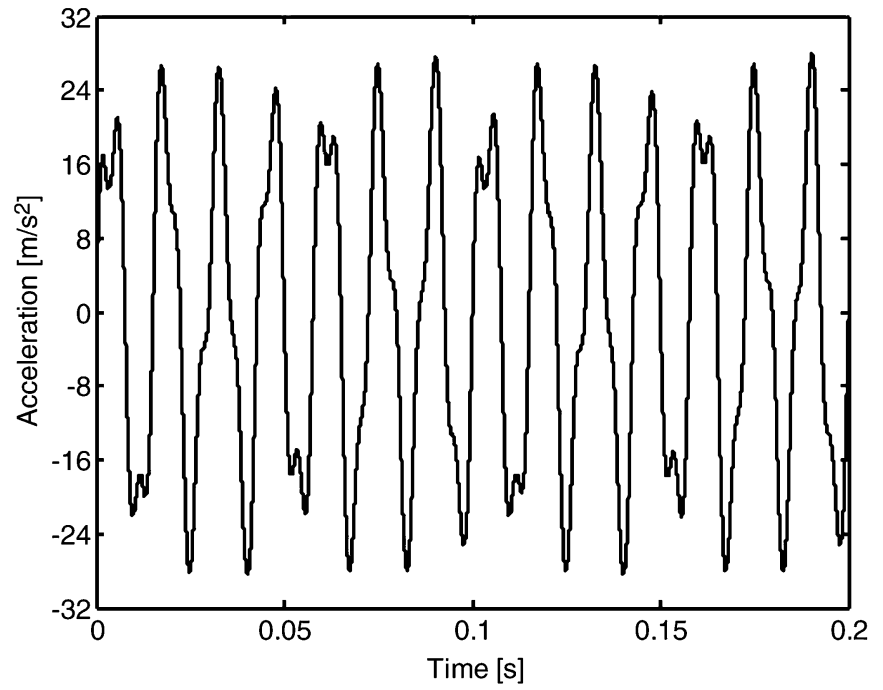

Fig. 7. Actual excitation acceleration with three components: 70, 110, and $190 \mathrm{~Hz}$.

for $\beta=0.5$. The expressions for the gains were normalized. In the case of the single accelerometer with harmonic excitation, the dc component was removed. Even if the modulation factor $\beta$ is large, the differential gain has a much lower level of distortion compared to the single-accelerometer case.

When the twin accelerometers actuated in quadrature are used, the differential output will contain a signal $\sim a_{\text {ext }}(t) \cos \left(2 \omega_{1} t\right)$, where $a_{\text {ext }}$ is the input acceleration, while $\omega_{1}$ is the frequency of the electrostatic driving force. An averaging filter will generate therefore the cosine spectral component of frequency $2 \omega_{1}$ present in the signal, $X c\left(2 \omega_{1}\right)$. A similar quadrature actuation, but with a phase difference of $\pi / 4$, will generate an output $\sim a_{\text {ext }}(t) \sin \left(2 \omega_{1} t\right)$. In such a case, the output of the averaging filter will correspond to the sine spectral component of frequency $2 \omega_{1}$ present in the input acceleration signal, $X s\left(2 \omega_{1}\right)$. As the phase shift between the input acceleration signal $\mathrm{a}_{\text {ext }}(\mathrm{t})$ and the time-varying actuation voltage is unknown, the amplitude of the spectral component of frequency $2 \omega_{1}$ is $\left|X\left(2 \omega_{1}\right)\right|=\sqrt{X_{c}^{2}\left(2 \omega_{1}\right)+X_{s}^{2}\left(2 \omega_{1}\right)}$.

A time-multiplexing method is used to extract the real and imaginary components, as shown in Fig. 4, of the desired spectral term $X\left(2 \omega_{1}\right)$ of the input mechanical vibration. By varying the frequency $\omega_{1}$ of the electrical actuation, the mechanical vibration spectrum is scanned.

\section{EXPERIMENTAL RESULTS}

For experimental validation, two prototype accelerometers were used (Fig. 2). The displacement is measured by sensing
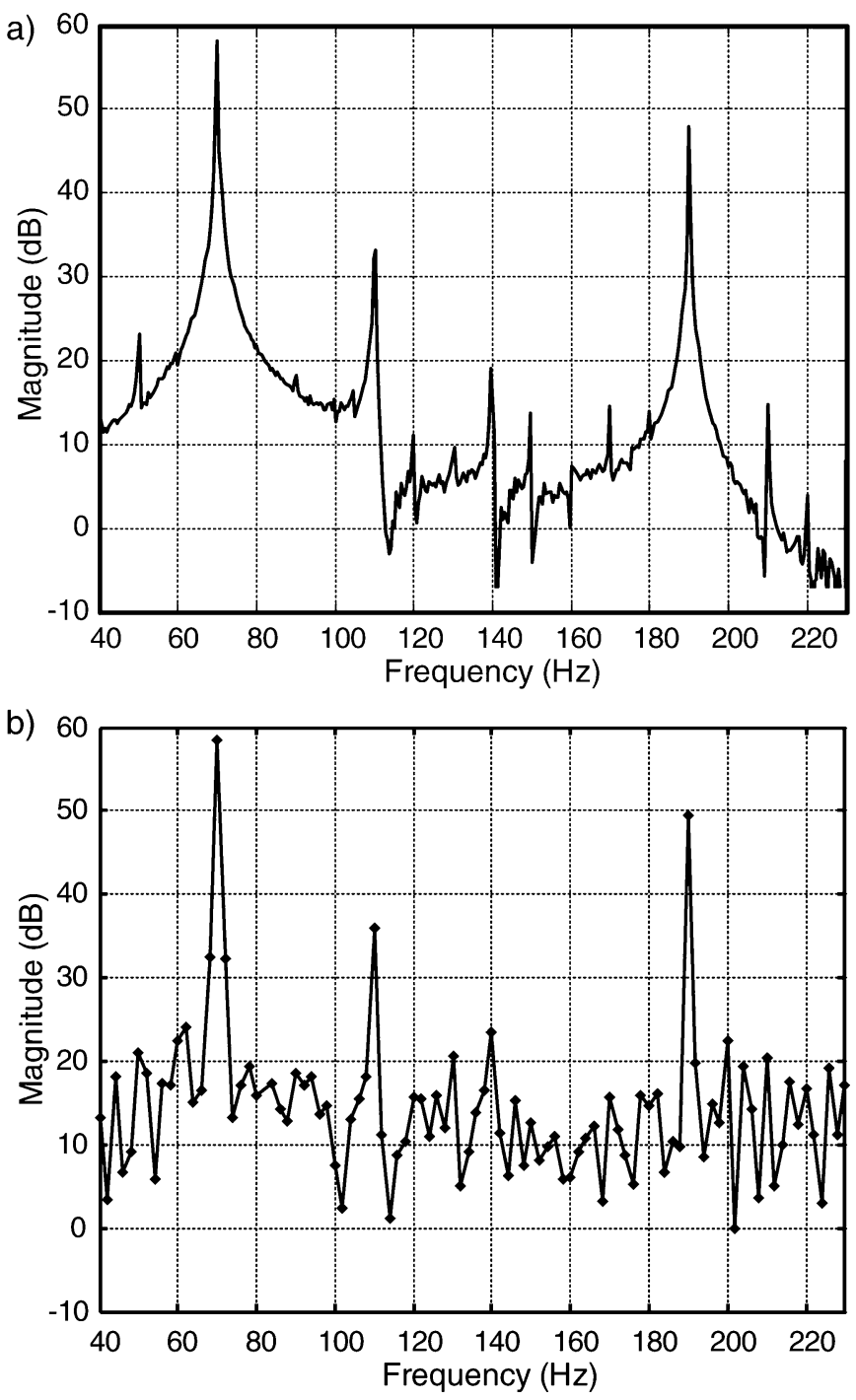

Fig. 8. Spectrum of a three-component input signal. (a) FFT. (b) Scanned spectrum.

the changes in a set of differential capacitors in the accelerometer. The use of a vibration table (Bruel \& Kjaer PM Vibration Exciter-Type 4808) allows us to generate the accelerations. To generate the desired waveforms an HP33120A function generator was used. By connecting the HP33120A output to the vibration table amplifier, the desired accelerations were applied to the accelerometers. The first acceleration test signal applied was composed of three components $(70,110$, and $190 \mathrm{~Hz})$, with 20 $\mathrm{dB}$ difference in peaks between the first and second component and $6 \mathrm{~dB}$ difference between the first and third component. The signal, with maximum amplitude of $27 \mathrm{~m} / \mathrm{s}^{2}$, was measured by one of the accelerometers in the absence of any electrostatic actuation and is presented in Fig. 7.

First, the output of the accelerometer in the absence of any electrostatic actuation was acquired with a $50 \mathrm{kHz}$ sampling rate, for $2 \mathrm{~s}$, and the FFT of the time series was computed. Basically, the FFT of the signal of Fig. 7 was computed and is presented in Fig. 8(a). The real-time spectrum extraction scheme (Fig. 4) was then applied, with a $2 \mathrm{~s}$ integration time for each point. The obtained spectrum is shown in Fig. 8(b). 


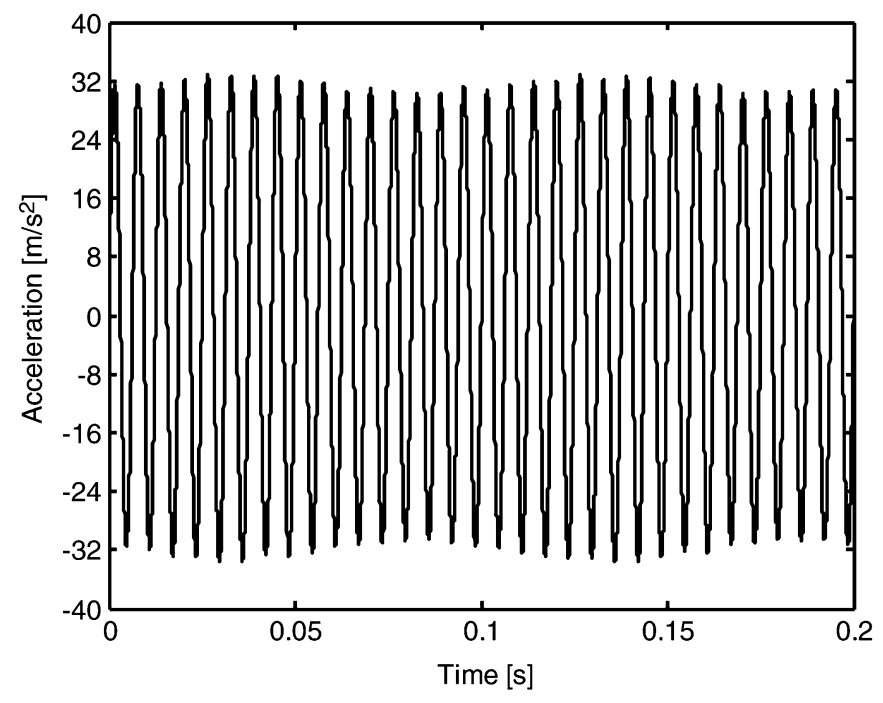

Fig. 9. Actual excitation acceleration with two closely spaced components: 160 and $170 \mathrm{~Hz}$.

In a second test, the spectrum of a signal with two closely spaced components and maximum amplitude of $32 \mathrm{~m} / \mathrm{s}^{2}$ was measured. The input signal, consisting of two components of 160 and $170 \mathrm{~Hz}$, respectively, and a $28 \mathrm{~dB}$ difference in peaks, was first acquired with a $50 \mathrm{kHz}$ sampling rate, for $4 \mathrm{~s}$, and no electrostatic actuation. It is presented in Fig. 9. The realtime scheme was then used, with an integration time of $4 \mathrm{~s}$. The comparative results are presented in Fig. 10 and again validate the concept.

The results obtained with the real-time spectrum scan compare well with FFT with the same acquisition time. Due to mismatches on the accelerometers, the noise level is higher in the proposed approach. Also some small delays introduced in the capacitive readouts of both accelerometers contribute to the oscillations observed at the lower level values. This complicates a comparison in terms of sensitivity.

The dynamic range depends on the choice of the nondimensional parameter $\beta$. A compromise among sensitivity, distortion level, and dynamic range has to be found in order to set the voltage amplitude used for electrostatic actuation. For higher sensitivities, the dynamic range decreases, while the distortion levels increase. In principle these values can be adjusted depending on the targeted application.

Similar to the FFT approach, the selectivity of the real-time spectrum scan depends on the integration (acquisition) time. In critical applications with closely spaced spectral peaks, the FFT performs better. However, the real-time spectrum analyzer is far more flexible when it comes to zooming-in.

\section{CONCLUSION}

The method presented here basically involves electronically controlled spectral filtering in the mechanical domain. The critical part is the compromise between achievable sensitivity and the distortion level, which determines the voltage amplitude used for electrostatic actuation.

Another important practical issue is the matching of the two accelerometers. However, any mismatch can be corrected by
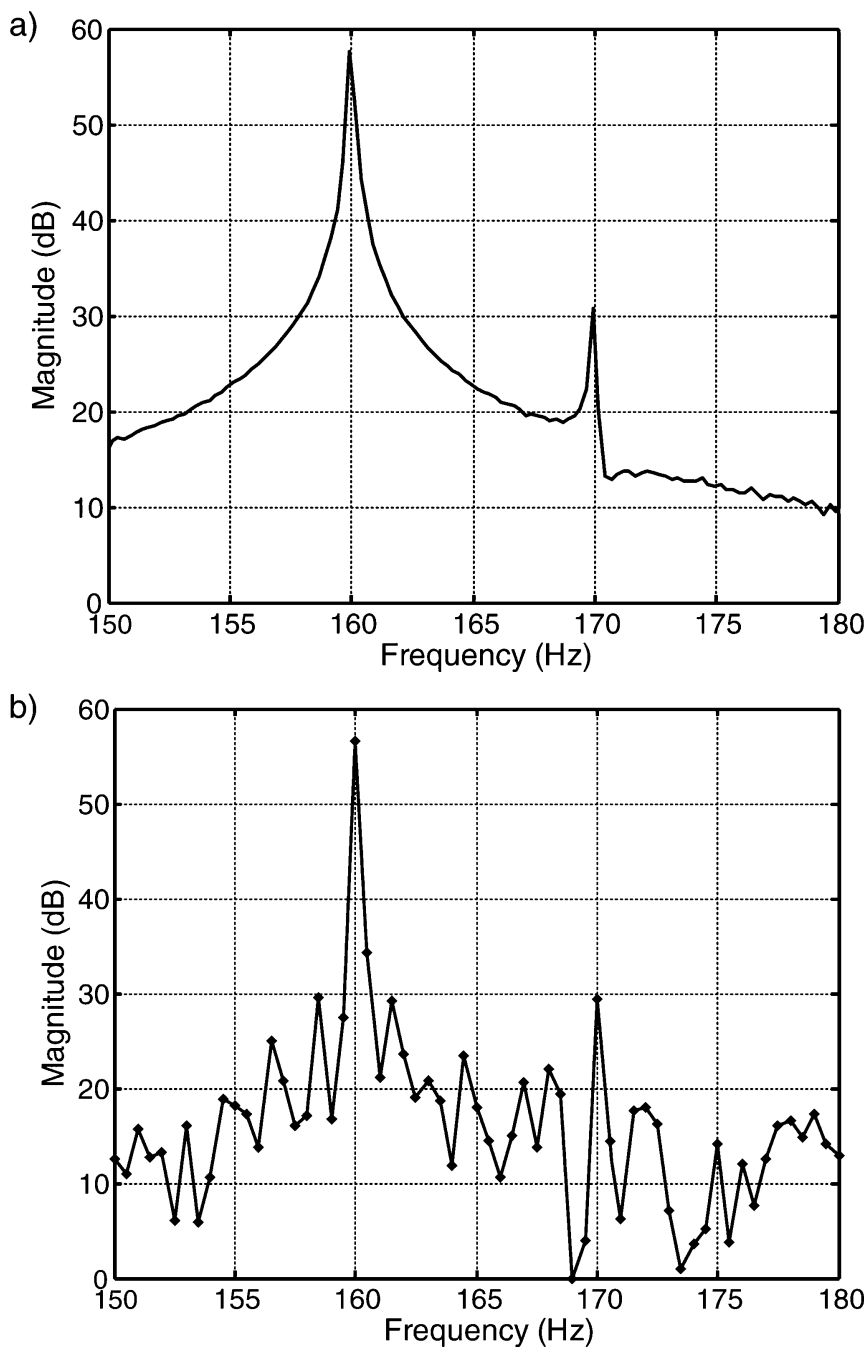

Fig. 10. Distinction between two closely spaced frequencies (160 and $170 \mathrm{~Hz})$ with $28 \mathrm{~dB}$ peak difference. (a) FFT. (b) Scanned spectrum.

using a different voltage amplitude for settling the sensitivity of the accelerometers.

First measurements indicate a dynamic range in excess of $40 \mathrm{~dB}$ and selectivity better than $10 \mathrm{~Hz}$ at $160 \mathrm{~Hz}$, at a $4 \mathrm{~s} \mathrm{ac}-$ quisition time. This performance compares well with FFT on a 4 s time series.

The capacitive transducer in combination with low-power readout circuits has the potential for lower power consumption compared to a digital system running an FFT algorithm. The method presented here is very suitable for low-power low-cost applications. This advantage becomes even more apparent in applications where, based on prior knowledge on failure mechanisms involved, one can decide to only monitor some known spectral components. This is often the case in condition monitoring systems.

\section{REFERENCES}

[1] G. T. A. Kovacs, Micromachined Transducers Sourcebook. Boston, MA: McGraw-Hill, 1998.

[2] C. Cempel, "Multifault condition monotoring of mechanical systems in operation," in Proc. IMEKO XVII, Croatia, 2003, pp. 1-4.

[3] R. A. Collacott, Vibration Monitoring and Diagnosis. London, U.K.: Gowdin, 1979. 
[4] M.-H. Bao, Micro Mechanical Transduce. Pressure Sensors, Accelerometers and Gyroscopes. Amsterdam, The Netherlands: Elsevier, 2000.

[5] E. Cretu, M. Bartek, and R. F. Wolffenbuttel, "Spectral analysis through electro-mechanical coupling," Sensors Actuators, vol. A85, no. 1-3, pp. 23-32, Aug. 2000.

[6] R. P. van Kampen and R. F. Wolffenbuttel, "Modeling the mechanical behavior of bulk-micromachined silicon accelerometers," Sensors Actuators, vol. A64, no. 2, pp. 137-150, Jan. 1998.

[7] R. Puers, M. Catrysse, G. Vandevoorde, R. J. Collier, E. Louridas, F. Burny, M. Donkerwolcke, and F. Moulart, "A telemetry system for the detection of hip prosthesis loosening by vibration analysis," in Proc. Eurosensors XIII, The Hague, the Netherlands, 1999, pp. 757-760.

[8] M. Offenberg, F. Lärmer, B. Elsner, H. Münzel, and W. Riethmüller, "Novel process for an integrated accelerometer" in Proc. Transducers'95, Stockholm, Sweden, 1995, pp. 589-593.

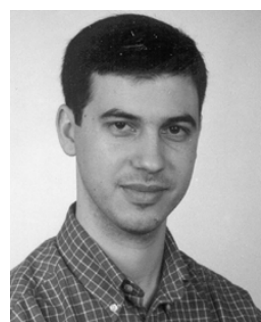

Luis Alexandre Rocha was born in Guimarães, Portugal, in 1977. In 1995, he began to study electronic engineering at the University of Minho, Portugal, where he graduated in 2000. Since February 2001, he has been pursuing the Ph.D. degree at the Department for Microelectronics, Faculty for Information Technology and Systems of the Delft University of Technology, Delft, The Netherlands. The topic of his research includes the study and design of MEMS for application in microinstruments.

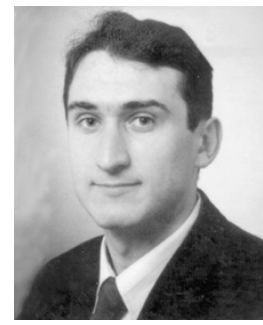

Edmond Cretu was born in Romania in 1965 . He received the M.Sc. degree in Electronic engineering from the polytechnic university of Bucharest in 1989 and the Ph.D. degree from Delft University of Technology, in 2003.

He was a Researcher at the Romanian Academy of Sciences and Associate Assistant at the Faculty of Electrical Engineering of the Polytechnic University of Bucharest. Since March 2000 he has been with Melexis Belgium, as Senior Designer and Project Manager in the field of inertial systems, with emphasis on MEMS-based gyroscope systems.

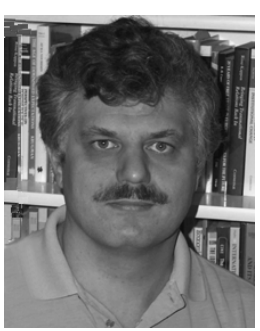

Reinoud F. Wolffenbuttel received the M.Sc. and $\mathrm{Ph} . \mathrm{D}$. degrees from Delft University of Technology, Delft, The Netherlands, in 1984 and 1988, respectively.

From 1986 to 1993, he has been an Assistant Professor, and since 1993, he has been an Associate Professor with the Department of Microelectronics, Faculty of Information Technology and Systems, Delft University of Technology, and is involved in instrumentation and measurement in general and on-chip functional integration of microelectronic circuits and silicon sensor, fabrication compatibility issues, and micromachining in silicon and microsystems in particular. He was a visitor at the University of Michigan, Ann Arbor, in 1992, 1999, and 2001, Tohoku University, Sendai, Japan, in 1995 , and EPFL Lausanne, Switzerland, in 1997. He served as General Chairman of the Dutch National Sensor Conference in 1996, Eurosensors in 1999, and the MicroMechanics Europe Workshop in 2003.

Dr. Wolffenbuttel is the recipient of a 1997 NWO pioneer award. 